\section{Socioeconomic deprivation and NHS orthodontic treatment delivery in Scotland}

\author{
A. Ulhaq, ${ }^{1}$ A. D. McMahon, ${ }^{2}$ S. Buchanan, ${ }^{3}$ S. Goold ${ }^{4}$ and D. I. Conway ${ }^{5}$
}

IN BRIEF
- Highlights that equity of access to
health services is a key marker of quality
healthcare services.
- Notifies that this is the first assessment
of the delivery of primary care NHS
orthodontic services across Scotland in
relation to area deprivation.
Informs that patients from most
deprived areas are less likely to receive
orthodontic treatment in the NHS
primary care setting in Scotland.

\begin{abstract}
Objective The purpose of this observational study was to investigate the relationship between deprivation and the delivery of primary care NHS orthodontic services across Scotland. Method Deprivation was measured using the Scottish Index of Multiple Deprivation (SIMD). The Information Services Division, NHS National Services Scotland, supplied data on all claims for orthodontic treatments in Scotland for the years 2008 and 2009. Each claim was assigned to a SIMD quintile (SIMD 1 being the most deprived, and SIMD 5 the least deprived), and odds ratios were calculated. Results Uptake of orthodontic services is highest in the least deprived areas. Patients from the least deprived areas are nearly twice as likely to receive orthodontic treatment as those from the most deprived areas (odds ratio of 1.90 with a 95\% confidence interval (CI) 1.86 to 1.94). Conclusion Patients from more the most deprived backgrounds are less likely to receive orthodontic treatment than those from more affluent backgrounds, which does not necessarily reflect need.
\end{abstract}

\section{INTRODUCTION}

Under the regulations of the NHS General Dental Service (GDS) in Scotland (which accounts for over 90\% of all NHS dental activity in Scotland), orthodontic care is available free to children. In the year ending March 2010, over £14 million was spent on orthodontic treatment for those under 18 years of age in the GDS in Scotland. ${ }^{1}$ The average cost of a course of dental treatment was around £60 per child, with $65 \%$ of the costs for treatment items of service attributed to orthodontic treatments. ${ }^{1}$ With increased focus on equity of access, and in the current economic climate, it is especially important to ensure that those in need of treatment are able to receive the services available. The Scottish Government have aimed to improve service provision in areas of unmet need by giving financial support (via the Scottish

\footnotetext{
1*Specialty Registrar in Orthodontics, East of England Deanery; ${ }^{2}$ Reader in Epidemiology, University of Glasgow Dental School; ${ }^{3}$ Senior Information Analyst, Information Services Division, NHS National Services Scotland; ${ }^{4}$ Principal Information Analyst, Information Services Division, NHS National Services Scotland; ${ }^{5}$ Clinical Senior Lecturer in Dental Public Health, University of Glasgow Dental School

*Correspondence to: Aman Ulhaq

Email:aman.ulhaq@kcl.ac.uk
}

Online article number E5

Refereed Paper - accepted 4 May 2012

DOI: 10.1038/sj.bdj.2012.724

${ }^{\circ}$ British Dental Journal 2012; 213: E5
Dental Access Initiative scheme grants) to practitioners, including orthodontic practitioners, in order to help establish or expand practices in these areas. ${ }^{2}$ The recent introduction of the Index of Orthodontic Treatment Need (IOTN) as a means of determining appropriate need and provision of orthodontic care in the NHS GDS statement of dental remuneration in Scotland ${ }^{3}$ has brought into sharp focus the issue of rationing limited resources particularly in relation to orthodontic care. From 1 October 2011, the contract has changed to one based on need with only those with moderate to severe malocclusion covered by NHS provision. ${ }^{3}$

Equity of access to health services is recognised as a key marker of quality of healthcare, which has been adopted by the NHS in Scotland. ${ }^{4}$ The 2003 UK Children's Dental Health Survey demonstrated that malocclusion was distributed evenly among 12-year-old children irrespective of gender or socioeconomic class. ${ }^{5}$ However, it also found that among 15-year-old children there was greater unmet need in children from more deprived schools.

Deprivation and its influence on orthodontic treatment delivery has been investigated in previous studies. The findings have been variable, with some showing that children from more deprived communities are less likely to receive orthodontic care, ${ }^{6,7}$ while others have found no link between deprivation and the uptake of orthodontic treatment provision. ${ }^{8,9}$ However, to date, none of these analyses has been undertaken in Scotland. The purpose of this study was to investigate the relationship between deprivation and delivery of primary care (GDS) NHS orthodontic services across Scotland.

\section{METHODS}

Deprivation was measured using the Scottish Index of Multiple Deprivation (SIMD), ${ }^{10}$ which identifies small areas of multiple deprivation across the whole of Scotland. Scotland is divided into 6,505 data zones ranked from the most deprived to the least deprived. The index combines information from seven domains which carry different weightings including: current income (28\%), employment (28\%), health (14\%), education (14\%), geographic access to services (9\%), crime (5\%), and housing (2\%). The SIMD assists in the targeting of policies and funding where the aim is to effectively tackle the problems associated with deprivation. The data zones are clustered into five deprivation quintiles with approximately 20\% of the Scottish population in each quintile. In accordance with SIMD 2009 notation, quintile 1 is the most deprived and quintile 5 the least deprived. 
The Information Services Division (ISD), NHS National Services Scotland (NSS), supplied data on all claims made for courses of orthodontic treatment for the financial years 2008 and 2009. The GP17(0) forms (orthodontic treatment claims) submitted to NSS Practitioner Services Division (PSD) for payment authorisation by the Scottish Dental Practice Board (SDPB) provided the necessary data for this study. These claims covered the whole of Scotland and were made by both dentists with list numbers restricted to the practice of orthodontics and general dental practitioners (GDPs) who undertake some orthodontic treatment. We analysed the data by both patient postcode (the patient's registered address), and dental surgery postcode (the location of the practice where the treatment was carried out). The analysis included patients who may have undergone an orthodontic assessment only; however, we have also provided data on those who received active appliance therapy. It is important to note that the data used were counts of individual patients and not the number of courses of treatment that they received.

To analyse the data, the total number of orthodontic claims was compared to the number of patients registered with GDPs to provide odds ratios for the SIMD quintiles. GDP registration figures were only available for 2010. Odds ratios (OR) and 95\% confidence intervals $(95 \% \mathrm{CI})$ were computed.

\section{RESULTS}

\section{Total claims}

In 2008, a total of 99,475 GP17(0) forms were paid. There were 2,869,205 adult patients registered and 869,000 children registered within the GDS Scotland. Of the total claims, 82,003 (82.4\%) were made by dentists with list numbers restricted to the practice of orthodontics. This figure increased slightly to 102,564 GP17(0) forms (an increase of 3.1\%) for 2009, with $87,283(85.1 \%)$ of these accounted for by dentists with list numbers restricted to the practice of orthodontics.

In relation to SIMD, quintiles 1 (most deprived) to 3 show a relatively even distribution; however, there was a much larger uptake of services in the least deprived proportion of the population (Fig. 1).

\section{Active appliances}

Only a fraction of the total claims $(17.6 \%$

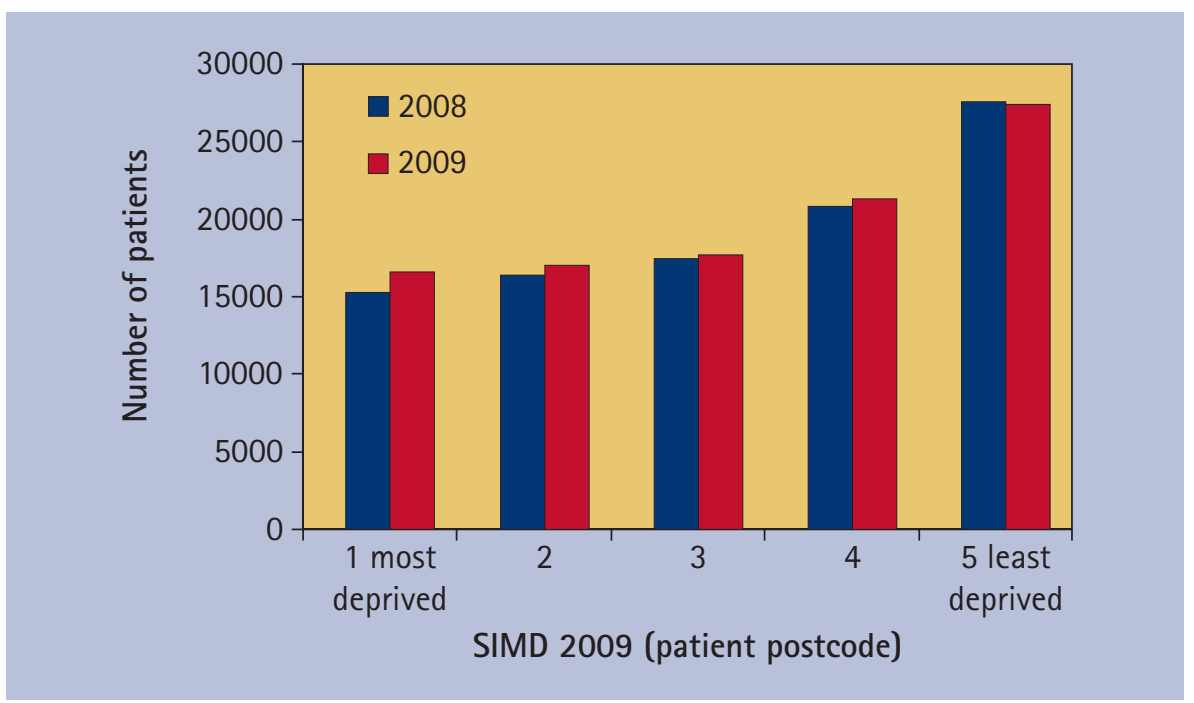

Fig. 1 Total number of claims for orthodontic treatment/assessment by GDPs and 'dentists with list numbers restricted to the practice of orthodontics' in relation to SIMD 2009

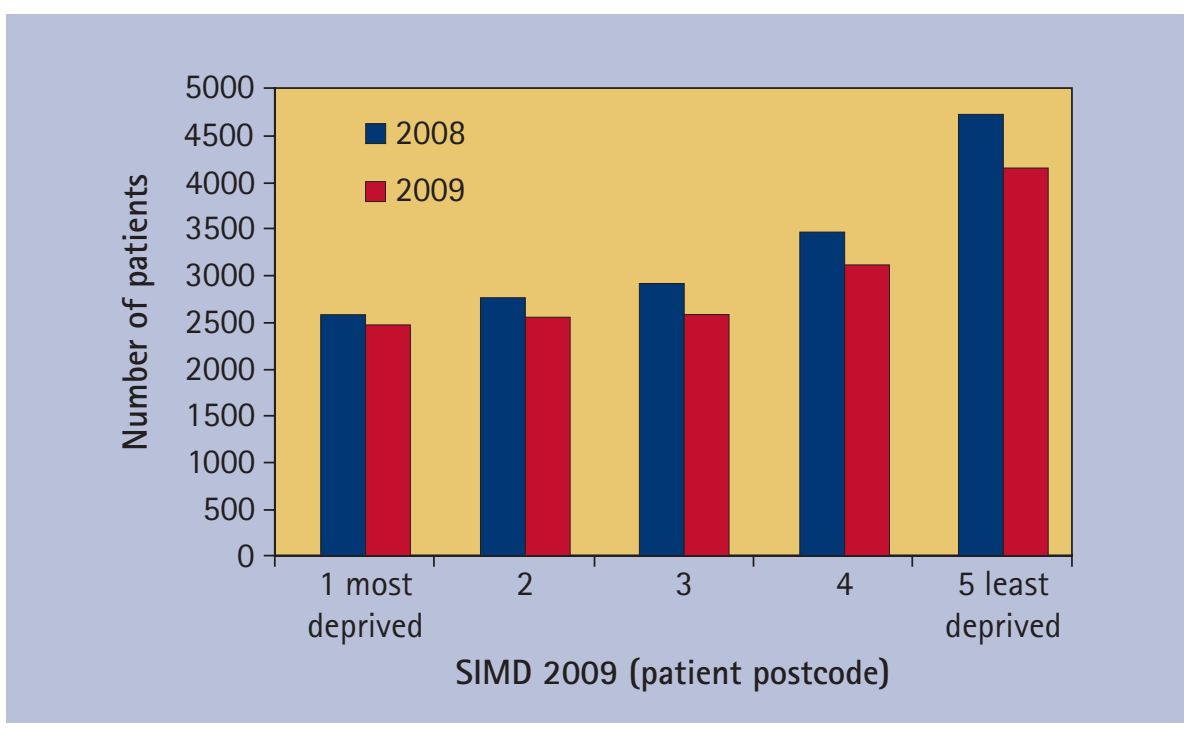

Fig. 2 Total number of patients provided with active orthodontic appliances by GDPs and 'dentists with list numbers restricted to the practice of orthodontics' in relation to SIMD 2009

in 2008 and $15.2 \%$ in 2009) made were for patients undergoing active appliance therapy. Our results don't take into account the type of appliance used, only the total number of patients who received treatment with an appliance. In 2008, 17,528 claims were made for orthodontic appliance therapy, 16,358 (93.3\%) of which were made by dentists with list numbers restricted to the practice of orthodontics. The total number of patients undergoing appliance therapy declined by $10.8 \%$ in the following year to 15,627 , and 14,721 of these patients (94.2\%) were treated by dentists with list numbers restricted to the practice of orthodontics.

When the figures are analysed according to SIMD quintiles, again we see an uneven distribution. The uptake of services is lower among the most deprived patients but higher again in SIMD quintiles 4 and 5 (Fig. 2).

\section{GDS registration}

Even though our findings show that registration rates for children are quite evenly distributed among the SIMD quintiles, the odds of patients from the most affluent areas (SIMD 5) receiving orthodontic treatment were nearly double those of patients from the most deprived areas (SIMD 1), with an OR of 1.90 in 2008 (95\% CI 1.86 to 1.94), declining only slightly in 2009 to OR 1.72 (95\% CI 1.62 to 1.76 ) (Table 1$)$.

The number of dental practices that are restricted to orthodontics increases with affluency of location, with the exception of SIMD 5. There is a steady increase in the distribution of these practices from SIMD 1 


\begin{tabular}{|c|c|c|c|c|c|c|}
\hline Year 2008 & No. treated & No. of treatments & ${ }^{*}$ No. registered & Percentage treated & Odds ratio & $95 \% \mathrm{Cl}$ \\
\hline SIMD 1 & 14,752 & 152,942 & 167,694 & 8.8 & & \\
\hline SIMD 2 & 15,825 & 142,575 & 158,400 & 9.99 & 1.15 & 1.12 to 1.18 \\
\hline SIMD 3 & 16,932 & 144,454 & 161,386 & 10.49 & 1.22 & 1.19 to 1.24 \\
\hline SIMD 4 & 20,284 & 149,322 & 169,606 & 11.96 & 1.41 & 1.38 to 1.44 \\
\hline SIMD 5 & 27,038 & 147,348 & 174,386 & 15.5 & 1.9 & 1.86 to 1.94 \\
\hline Year 2009 & No. treated & No. of treatments & ${ }^{*}$ No. registered & Percentage treated & Odds ratio & $95 \% \mathrm{Cl}$ \\
\hline SIMD 1 & 16,061 & 151,633 & 167,694 & 9.58 & & \\
\hline SIMD 2 & 16,553 & 141,847 & 158,400 & 10.45 & 1.1 & 1.08 to 1.13 \\
\hline SIMD 3 & 17,122 & 144,264 & 161,386 & 10.61 & 1.12 & 1.10 to 1.15 \\
\hline SIMD 4 & 20,769 & 148,837 & 169,606 & 12.25 & 1.32 & 1.29 to 1.35 \\
\hline SIMD 5 & 26,883 & 147,503 & 174,386 & 15.42 & 1.72 & 1.69 to 1.76 \\
\hline
\end{tabular}

to SIMD 4. SIMD 4 has nearly double the number of specialist orthodontic practices when compared with SIMD1.

\section{DISCUSSION}

This is the first study to demonstrate the relationship that exists between socioeconomic deprivation and the uptake of NHS orthodontic services in Scotland. We have used data at national level to provide a large sample. Our results indicate that those patients from more deprived areas of the country are less likely to receive the treatment that they may have a clinical need for.

It appears that practices limited to orthodontics are located more frequently in more affluent areas. This may partially explain that children from more affluent areas are nearly twice as likely to receive orthodontic treatment than those from more deprived areas.

There may be other factors involved that result in this inequity in NHS orthodontic services in Scotland. It is possible that patients are being referred for treatment but do not attend. There may also be a lack of awareness regarding orthodontic services among people from more deprived areas. However, if a lack of awareness is shown to exist, then it is the responsibility of GDPs and orthodontists to educate patients and ensure these patients know what services are available to them, and to refer them based on need.

GDPs are the main source of referral to dentists who restrict their practice to orthodontics, thus one would expect that registration rates could be an important factor for patients to go on and seek treatment or advice. In Scotland registration rates are fairly even across the SIMD quintiles and are unlikely to contribute negatively to orthodontic treatment uptake.

There is an important aspect of NHS orthodontic service provision that our data did not cover and we must interpret the results with some care; analysis here has been limited to data sourced from NHS general dental services and has not accounted for those patients seen in NHS hospital, community orthodontic services, or indeed privately (although we anticipate the vast majority of provision is in the GDS).

Furthermore, GDS registration figures were only available for the year 2010 whereas orthodontic claims data were available for the years 2008 and 2009. Although this will mean there was an element of prediction to calculate the odds ratios, the authors feel that there is unlikely to be a substantial difference in GDS registrations between these years.

The inclusion of a health domain in SIMD 2009 may have resulted in mathematical coupling as there is a measure of health on both sides of the correlation equation. Mathematical coupling can lead to erroneous results. ${ }^{11}$ However, Adams et al. ${ }^{12}$ previously showed that removing the health domain from the Index of Multiple Deprivation 2004 had little effect on measured socioeconomic inequalities in health.

\section{CONCLUSION}

There is a greater likelihood of patients from affluent areas receiving NHS orthodontic services in Scotland. The relationship between orthodontic treatment uptake and treatment need appears to be a complex one, although this finding is likely to be an example of the inverse care law - whereby orthodontic care is not based on need and not necessarily equitably accessed. There are likely to be many factors at the level of individual patients and their families that affect these decisions. We have only touched on this and there is a need for further research in this area. Our work supports the recent decision to begin to move the provision of NHS orthodontic services in Scotland to a service based on orthodontic need.

1. NHS Scotland. Scottish Dental Practice Board annual report 2009/10. Edinburgh: NHS National Services Scotland.

2. The Scottish Government. Revised Scottish Dental Access Initiative. Edinburgh: The Scottish Government, 2008.

3. Health and Social Care Integration Directorate NHS: PCA(D)(2011) 6. Orthodontic treatment - introduction of Index of Orthodontic Treatment Need. Edinburgh: Scottish Government, 2011.

4. The Scottish Government. The healthcare quality strategy for NHS Scotland. Edinburgh: The Scottish Government, 2010.

5. Office for National Statistics. National children's dental health survey. London: ONS, 2003.

6. Morris E, Landes D. The equity of access to orthodontic dental care for children in the North East of England. Public Health 2006; 120: 359-363.

7. Drugan C S, Hamilton S, Naqvi H, Boyles J R. Inequality in uptake of orthodontic services. Br Dent J 2007; 202: E15.

8. O'Brien $\mathrm{K}, \mathrm{McC}$ Comb J L, Fox N, Wright J. Factors influencing the uptake of orthodontic treatment. Br J Orthod 1996; 23: 331-334.

9. Breistein B, Burden D J. Equity and orthodontic treatment: a study among adolescents in Northern Ireland. Am J Orthod Dentofacial Orthop 1998; 113: 408-413.

10. The Scottish Government. The Scottish Index of Multiple Deprivation: 2009 general report Edinburgh: The Scottish Government, 2009.

11. Archie JP Jr. Mathematic coupling of data: a common source of error. Ann Surg 1981; 193: 296-303.

12. Adams J, White M. Removing the health domain from the Index of Multiple Deprivation 2004 effect on measured inequalities in census measure of health. J Public Health 2006; 28: 379-383. 